\title{
Addendum: A regulated PNUTS mRNA to IncRNA splice switch mediates EMT and tumour progression
}

\author{
Simon Grelet, Laura A. Link, Breege Howley, Clémence Obellianne, Viswanathan Palanisamy, \\ Vamsi K. Gangaraju, J. Alan Diehl and Philip H. Howe
}

Nature Cell Biology 19, 1105-1115 (2017); published online 19 July 2017; corrected after print 13 November 2017.

In this Addendum, the authors include western blot data using a C-terminal PNUTS antibody. This is important in that an annotation of the alternative spliced form of PNUTS, denoted in the UCSC genome browser (https://genome.ucsc.edu/), depicts it as a non-coding RNA. However, downstream of the alternative splice site is an alternative AUG located in frame in the PNUTS ORF at position 1039. The potential for a protein product of $\sim 61 \mathrm{kDa}$ being generated from this AUG was examined experimentally using a C-terminal raised antibody to PNUTS to exclude the possibility that the N-terminal deletion of the splice isoform was not the reason that the predicted 61 -kDa protein was not detected in cells using an $\mathrm{N}$-terminal generated antibody. The results presented here confirm our previous results using the N-terminal PNUTS antibody and originally presented in Supplementary Fig. $2 \mathrm{~b}$ of the Article; namely, that this predicted $\sim 61-\mathrm{kDa}$ product is not detectable in cells under the conditions used, even under conditions of overexpression.

Figure: IncRNA-PNUTS does not encode for a N-terminal truncated-protein product. The result of a western blot analysis of PNUTS protein expression in CaCo-2 cells upon transient lncRNA-PNUTS expression (3 days) or TGFß treatment ( 1 day) is shown. The C-terminal antibody used was EPR11706 (Abcam: Ab173285; clone PPP1R10; 1/1000 dilution) raised against the C-terminal region of the PNUTS protein (amino acids 550-650). The western blot protocol and extracts used in this experiment were identical to those described in Supplementary Fig. 2 of the original Article.

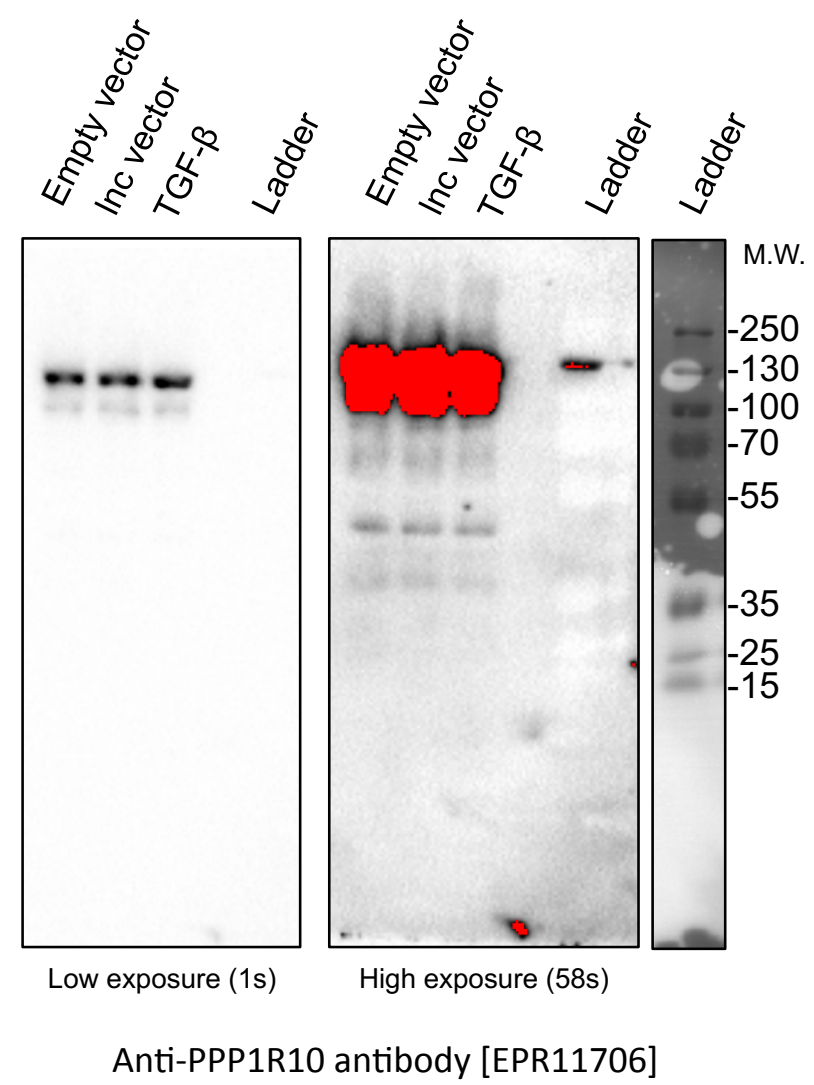

